\title{
Ultrafast Dynamics of a Nucleobase Analogue Illuminated by a Short Intense X-ray Free Electron Laser Pulse
}

K. Nagaya, ${ }^{1,2}$ K. Motomura, ${ }^{3}$ E. Kukk, ${ }^{4}$ H. Fukuzawa, ${ }_{5}^{2,3}$ S. Wada, ${ }^{2,5}$ T. Tachibana, ${ }^{3}$ Y. Ito, ${ }^{3}$ S. Mondal, ${ }^{3}$ T. Sakai, ${ }^{1}$ K. Matsunami, ${ }^{1}$ R. Koga,${ }^{5}$ S. Ohmura, ${ }^{1,6}$ Y. Takahashi, ${ }^{7}$ M. Kanno, ${ }^{7}$ A. Rudenko, ${ }^{8}$ C. Nicolas, ${ }^{9}$ X.-J. Liu, ${ }^{9,10}$ Y. Zhang, ${ }^{11}$ J. Chen, ${ }^{12}$ M. Anand, ${ }^{13,14}$ Y. H. Jiang, ${ }^{11}$ D.-E. Kim, ${ }^{13,14}$ K. Tono, ${ }^{15}$ M. Yabashi, ${ }^{2,15}$ H. Kono, ${ }^{7}$ C. Miron, ${ }^{9,16}$ M. Yao, ${ }^{1}$ and K. Ueda ${ }^{2,3}$

${ }^{1}$ Department of Physics, Graduate School of Science, Kyoto University, Kyoto 606-8502, Japan

${ }^{2}$ RIKEN Spring-8 Center, Sayo, Hyogo 679-5148, Japan

${ }^{3}$ Institute of Multidisciplinary Research for Advanced Materials, Tohoku University, Sendai 980-8577, Japan

${ }^{4}$ Department of Physics and Astronomy, University of Turku, FI-20014 Turku, Finland

${ }^{5}$ Department of Physical Science, Hiroshima University, Higashi-Hiroshima 739-8526, Japan

${ }^{6}$ Research Center for Condensed Matter Physics, Hiroshima Institute of Technology, Hiroshima 731-5193, Japan

${ }^{7}$ Department of Chemistry, Graduate School of Science, Tohoku University, Sendai 980-8578, Japan

${ }^{8}$ J.R. Macdonald Laboratory, Department of Physics, Kansas State University, 66506 Manhattan, Kansas, USA

${ }^{9}$ Synchrotron SOLEIL, L'Orme des Merisiers, Saint-Aubin, BP 48, FR-91192 Gif-sur-Yvette Cedex, France

${ }^{10}$ School of Physics and Nuclear Energy Engineering, Beihang University, Beijing 100191, China

${ }^{11}$ Shanghai Advanced Research Institute, Chinese Academy of Sciences, 201210 Shanghai, China

${ }^{12}$ Shanghai Institute of Applied Physics, Chinese Academy of Sciences, 201800 Shanghai, China

${ }^{13}$ Department of Physics, Center for Attosecond Science and Technology, Pohang University of Science and Technology, Pohang, 37673, South Korea

${ }^{14}$ Max Planck Center for Attosecond Science, Max Planck POSTECH/KOREA Research Initiative, Pohang 37673, South Korea

${ }^{15}$ Japan Synchrotron Radiation Research Institute (JASRI), Sayo, Hyogo 679-5198, Japan

${ }^{16}$ Extreme Light Infrastructure-Nuclear Physics (ELI-NP), "Horia Hulubei" National Institute for Physics and Nuclear Engineering, 30 Reactorului Street, RO-077125 Măgurele, Judetul Ilfov, Romania

(Received 4 April 2016; revised manuscript received 18 May 2016; published 16 June 2016)

Understanding $\mathrm{x}$-ray radiation damage is a crucial issue for both medical applications of $\mathrm{x}$ rays and $\mathrm{x}$-ray free-electron-laser (XFEL) science aimed at molecular imaging. Decrypting the charge and fragmentation dynamics of nucleobases, the smallest units of a macro-biomolecule, contributes to a bottom-up understanding of the damage via cascades of phenomena following x-ray exposure. We investigate experimentally and by numerical simulations the ultrafast radiation damage induced on a nucleobase analogue (5-iodouracil) by an ultrashort (10 fs) high-intensity radiation pulse generated by XFEL at SPring-8 Angstrom Compact free electron Laser (SACLA). The present study elucidates a plausible underlying radiosensitizing mechanism of 5-iodouracil. This mechanism is independent of the exact composition of 5-iodouracil and thus relevant to other such radiosensitizers. Furthermore, we found that despite a rapid increase of the net molecular charge in the presence of iodine, and of the ultrafast release of hydrogen, the other atoms are almost frozen within the 10-fs duration of the exposure. This validates singleshot molecular imaging as a consistent approach, provided the radiation pulse used is brief enough.

DOI: 10.1103/PhysRevX.6.021035

\section{INTRODUCTION}

During the last decade, new research avenues have been opened for both spectroscopic and structural investigations of matter, through the advent of the x-ray free-electronlaser (XFEL) [1-3]. XFELs deliver coherent $\mathrm{X}$-ray pulses,

Published by the American Physical Society under the terms of the Creative Commons Attribution 3.0 License. Further distribution of this work must maintain attribution to the author(s) and the published article's title, journal citation, and DOI.
Subject Areas: Atomic and Molecular Physics

combining unprecedented intensities and extremely short pulse durations, as short as a few femtoseconds only. For selected applications, these qualities offer important advantages over conventional $\mathrm{x}$-ray sources. Time-resolved spectroscopy on the time scale of femtoseconds to picoseconds allows us to monitor, for the first time, atoms and electrons in action [4-14]. For structural analysis, the extreme intensity focused into a couple of $\mu \mathrm{m}^{2}$ transforms single-shot diffraction imaging of biomolecules and nanosize objects from a remote goal into a tangible reality [15-19].

Connecting these two realms of XFEL-based research is the fundamental issue of radiation damage. X-ray 
absorption often leads to the breakage of chemical bonds, geometrical changes, and distortions of electron distributions that manifest the deterioration of the original object. In XFEL-based experiments for a single-molecule or a nanosize object, the amount of energy deposited in the target by a single pulse is sufficiently high to cause its total destruction; from a structural imaging point of view, the question is whether the time scale of these changes is slow enough for the collected data to capture essential highquality structural information about the original object [20]. Our present study addresses this question.

The phenomenon of radiation damage is also interesting by itself because it is a consequence of the complex interplay between fast electron dynamics and slower nuclear dynamics, following the rapid creation of highly charged, highly energetic states. X-ray radiation creates charges by core ionization, which is followed by internal electronic relaxations, i.e., Auger processes that may occur in cascades. Core ionization is thus predominantly the first step of radiation damage in biomolecules from living tissue subjected to medical or unintentional x-ray exposure [21]. The cascading processes of creating low-energy electrons and ionic radicals, which play a crucial role in the damage, are also of current fundamental interest [22,23]. XFEL pulses, which are the subject of the present work, are known to cause, even for isolated atoms, major changes in electronic structure via multiphoton absorption followed by Auger processes [24-27]. At the other end of the scale, in condensed matter and clusters of $10^{3}-10^{5}$ atoms, the intense XFEL pulses produce a dense nanoplasma, in which the dynamics of electrons and ions are strongly correlated with each other $[28,29]$.

The coupled electron and ion dynamics strongly depend on the size of the system, laser fluence, and photon energy. Studying the response of a single molecule composed of a small number of atoms to the XFEL pulse is perhaps the most rewarding, as various levels of theory and experimental methods inapplicable to larger systems may be applied. This was illustrated by Erk et al., who reported ionization and fragmentation of methylselenol $\left(\mathrm{CH}_{3} \mathrm{SeH}\right)$ molecules by intense $\left(>10^{17} \mathrm{~W} / \mathrm{cm}^{2}\right)$ 5-fs long x-ray pulses of about $2 \mathrm{keV}$ energy, using coincident ion momentum spectroscopy [30]. They reported a molecular expansion of about $50 \%$ within the first 5-10 fs. Motomura et al. reported a similar study on iodomethane $\left(\mathrm{CH}_{3} \mathrm{I}\right)$ molecules using XFEL pulses of 5.5 keV at SACLA XFEL in Japan [31]. The latter study concluded that the C-I bond length increased by only $10 \%$ during the first 10 fs.

The target of the present study is a more complex molecule, a 5-halouracil, which is an analogue of uracil, one of the four canonical nucleobases of ribonucleic acid (RNA). While 5-fluorouracil (FU) was identified several decades ago as an effective anticancer drug [32], iodine substitution makes the uracil molecule much more vulnerable to $\mathrm{x}$-ray damage owing to iodine's large absorption cross section for hard $\mathrm{x}$ rays. Indeed, a possible use of 5-iodouracil (IU) as a radiosensitizer in tumor therapy has been discussed [33] and the radiation damage involving 5-IU has been studied extensively by simulations [34,35]. To our knowledge, however, there has been no experimental report on the destruction of 5-IU exposed to x rays.

The aim of this work is to trace back the intricate details of the early stages of the Coulomb explosion of 5-IU, as a biomolecule of practical relevance, utilizing XFEL pulses as the ionizing radiation and advanced multiparticle coincidence detection techniques as an analysis tool. The experiment is interpreted with an improved version of our modeling approach elaborated for the first time with iodomethane [31]. The present results provide the basis for the understanding of the molecular mechanisms of the radiosensitizing capacity of 5-IU or any similar radiosensitizing molecules, as well as the basis for single-shot bio-imaging with XFEL.

\section{RESULTS}

Here, we report the results of the ion momentum imaging measurements for 5-IU using the intense x-ray pulses at the SACLA XFEL facility. The fragment ions resulting from the destruction of the sample, by multiphoton $\mathrm{x}$-ray absorption, were detected by a multicoincidence recoil ion momentum spectrometer [36,37] [see Fig. 1(a)], which provides the mass-to-charge ratios of all detected ions (via their flight time toward the detector), as well as the full reconstruction of their three-dimensional momenta. Let us first review the fragmentation pattern of 5-IU. Figure 1(b) shows the ion time-of-flight (TOF) spectrum of 5-IU, where it can be seen that (i) atomic fragments dominate the spectrum, (ii) doubly charged ions such as $\mathrm{C}^{2+}, \mathrm{O}^{2+}$, $\mathrm{N}^{2+}$, as well as multiply charged iodine ions $\mathrm{I}^{q+}$ $(q=1-4)$, are formed, and (iii) singly charged polyatomic fragments, $\mathrm{CC}^{+}, \mathrm{CN}^{+}, \mathrm{CO}^{+}, \mathrm{CNH}^{+}, \mathrm{COH}^{+}$, are also present, albeit with quite small abundances. The x-ray energy of $5.5 \mathrm{keV}$ used in this experiment can ionize electrons from and above the $\mathrm{L}$ shell of iodine with a much higher cross section than those from the $\mathrm{K}$ shells of light elements, making iodine the dominant $\mathrm{x}$-ray absorber in 5 -IU.

Regarding x-ray absorption, iodine behaves very similarly to its neighboring element xenon, where a prior XFEL experiment saw the creation of charge states up to +26 [27]. In the case of the smallest iodine-containing organic molecule, $\mathrm{CH}_{3} \mathrm{I}$, iodine ions up to $q=+15$ and carbon ions up to $q=+4$ were observed at similar XFEL conditions [31]. The significantly lower charge states of iodine and carbon atomic fragments from the larger molecule 5-IU suggest a very efficient redistribution [38] of the charges from the iodine to the entire molecule, over many more constituent atoms.

The dynamics of the absorption, charge redistribution, and early-stage fragmentation leaves an imprint on the final 
(a)

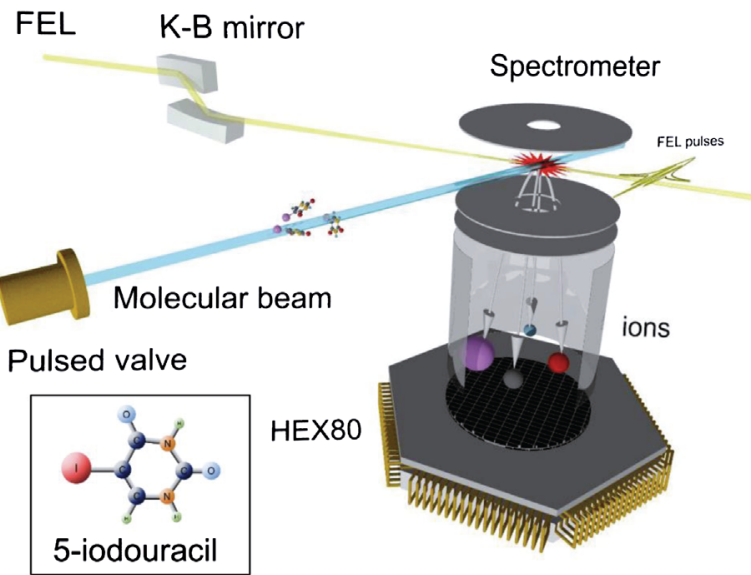

(b)

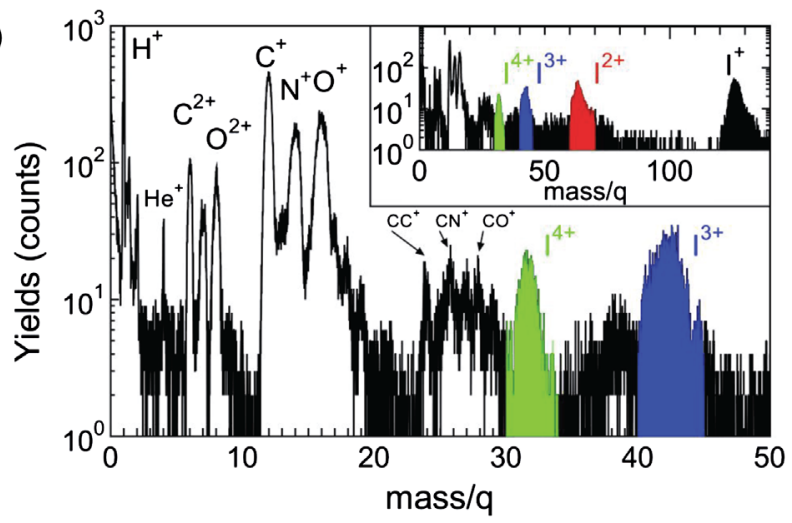

FIG. 1. Schematics of experimental setup and ion TOF spectrum. (a) Schematics of experimental setup. Molecular structure of 5-iodouracil is shown in the inset. (b) Yields of the 5-iodouracil ion TOF spectrum, recorded at $5.5 \mathrm{keV}$, are shown on a log scale and are plotted as a function of mass-to-charge ratio. The inset displays the full-range TOF spectrum.

momentum vectors of the fragment ions. Figure 2 represents this information in a compact form, as the kinetic energy distributions (KEDs) of the fragment ions derived from their observed momenta. Each dissociating 5-IU molecule results in several charged fragments; these were detected in coincidence with each other, allowing us to distinguish processes that produce, for example, $\mathrm{C}^{+}$combined with $\mathrm{I}^{+}$, or $\mathrm{C}^{+}$together with the $\mathrm{I}^{4+}$ ions.

In the kinetic energy analysis, the momentum correlation of the coincident ions is not revealed, but let us consider the angles formed between the momenta of the atomic fragments in the Coulomb explosion. The cosine of the $\theta$ angle, between the momenta of the two ionic fragments $\mathrm{A}$ and $\mathrm{B}$, is

$$
\cos (\theta)=\frac{\overrightarrow{p_{A}} \cdot \overrightarrow{p_{B}}}{\left|\overrightarrow{p_{A}}\right|\left|\overrightarrow{p_{B}}\right|}
$$

We denote the distributions of $\cos (\theta)$ between the momenta of various ejected ion pairs as $\mathrm{SP}_{2}(\mathrm{~A}, \mathrm{~B})$, and they are shown in the left column of Fig. 3. The observed maxima in the distributions $\mathrm{SP}_{2}\left(\mathrm{I}^{q+}, \mathrm{H}^{+}\right), \mathrm{SP}_{2}\left(\mathrm{I}^{q+}, \mathrm{O}^{+}\right)$, and $\mathrm{SP}_{2}\left(\mathrm{I}^{q+}, \mathrm{N}^{+}\right)$ correspond well to the angles in the equilibrium geometry of the parent 5-IU molecule, and they are insensitive to the charge state of the coincident iodine. In contrast, $\mathrm{SP}_{2}\left(\mathrm{I}^{+}, \mathrm{C}^{+}\right)$ has a maximum at around $60^{\circ}$, which tends to disappear when the iodine charge state increases and the angle between iodine and carbon ions becomes larger.

During the Coulomb explosion of 5-IU, almost always more than two ions are created. Up to now, we have studied them in pairs. Investigating triple coincidences allows us to extract even more detailed information on momentum correlations. In order to see how well the momenta of three ions $\mathrm{A}, \mathrm{B}$, and $\mathrm{C}$, lie on a molecular plane, a triple product was calculated from the observed momenta, giving the cosine of the polar angle:

$$
\cos (\phi)=\frac{\left(\overrightarrow{p_{A}} \times \overrightarrow{p_{B}}\right) \cdot \overrightarrow{p_{C}}}{\left|\overrightarrow{p_{A}} \times \overrightarrow{p_{B}}\right|\left|\overrightarrow{p_{C}}\right|}
$$

The experimental distributions, denoted as $\mathrm{SP}_{3}(\mathrm{~A}, \mathrm{~B}, \mathrm{C})$, are shown in the left column of Fig. 4. Note that the distribution function $\mathrm{SP}_{3}$ for three randomly oriented and uncorrelated vectors is flat against $\cos (\phi)$, so the curves presented directly reflect the deviation from isotropic emission. Coulomb explosion that occurs preferentially in the plane then results in the distributions of $\mathrm{SP}_{3}(\mathrm{~A}, \mathrm{~B}, \mathrm{C})$ peaked at $\cos (\phi)=0$. In Fig. 4, we chose the combinations $\mathrm{SP}\left(\mathrm{I}^{q+}, \mathrm{H}^{+}, \mathrm{x}\right)$, where $\mathrm{x}$ stands for the $\mathrm{H}^{+}, \mathrm{O}^{+}, \mathrm{N}^{+}$, or $\mathrm{C}^{+}$. $\mathrm{I}^{q+}, \mathrm{H}^{+}, \mathrm{N}^{+}$, and $\mathrm{O}^{+}$tend to be ejected preferentially in the plane, but this is not necessarily the case for $\mathrm{C}^{+}$.

\section{DISCUSSIONS}

In order to interpret the experimental results and quantify the underlying dynamics, we carried out classical MD simulations of the Coulomb explosion within a parametric model of charge buildup and redistribution. The model follows what was developed for the analysis of the $\mathrm{CH}_{3} \mathrm{I}$ molecule [31], keeping up with its original purpose of being easily extendable to various systems. The model appropriately considers the effects of multiple photon absorption within the XFEL pulse duration (about $10 \mathrm{fs}$ ) by the deep atomic shells of iodine. Concomitant to this absorption, but also partly subsequent to it, Auger cascades are the dominant mechanism of charge multiplication at an exponentially decreasing rate. We modeled these charge-creation processes statistically by assuming an increase of the total charge of the molecule $\left(Q^{\text {tot }}\right)$ with time $t$ (measured from the onset of the XFEL pulse) as $Q^{\mathrm{tot}}[t]=Q^{\max }[1-\exp (-t / \tau)]$, where $Q^{\max }$ is the final charge. Charge redistribution from iodine to the uracil ring $\left(\mathrm{C}_{4} \mathrm{H}_{3} \mathrm{~N}_{2} \mathrm{O}_{2}\right)$ is described by the rate equation $d Q^{\text {ring }}[t] / d t=R Q^{\mathrm{I}}[t]$, with a constant charge redistribution rate $R$. Here, $Q^{\text {ring }}[t]$ and $Q^{\mathrm{I}}[t]$ represent the charge of the uracil ring and iodine at $t$, respectively. This model describes the initial charge creation and the subsequent charge redistribution over the molecule as a result of, e.g., 
molecular Auger decay that occurs during the later stages of the Auger cascades. The two empirical constants $\tau$ and $R$ can be adjusted to best describe the experimental results.

As an important development of our earlier model, we now include molecular vibrations as dependent on the internal temperature $T$. First, molecules with randomly selected atomic velocities were prepared. Then, ion trajectories in the Coulomb explosion were obtained numerically using Coulomb repulsion forces between ions, which are regarded as point charges with the charge development depicted by the above model. The effect of covalent bonding in the 5-IU molecule was neglected. Thus, the di- and polyatomic fragments were not taken into account in the simulation. As shown in Fig. 1(b), complete fragmentation into atomic ions is the dominant process. A large number (1000 runs for each $Q^{\max }$ ) of trajectories were simulated for each set of parameter combinations of $\tau$, $R$, and $T$, and for a given charge $Q^{\max }$, differing by the random set of initial atomic velocities. Thousands of different sets of initial conditions afforded simulated statistical distributions, which can be directly comparable with the experimental ones, as seen in Figs. 2-4.

As seen in Fig. 2, the experimentally observed broadening of KEDs is well reproduced by the MD simulations (red solid lines). The MD simulations confirm that the broad widths of KED mainly come from the different charge distributions on atoms. The results of the MD simulations for KEDs without consideration of the effect of charge buildup and charge redistribution are shown in Fig. 2 by blue dotted lines. One can see that these simplified simulations fairly reproduce the experimental KEDs of $\mathrm{O}^{+}$ and $\mathrm{N}^{+}$, although they provide discrepancies for $\mathrm{H}^{+}$and $\mathrm{C}^{+}$, where they overestimate the kinetic energies. In the case of $\mathrm{H}^{+}$, this discrepancy can be directly attributed to the neglect of charge buildup time; in reality, the very mobile protons escape before the full charge buildup is reached
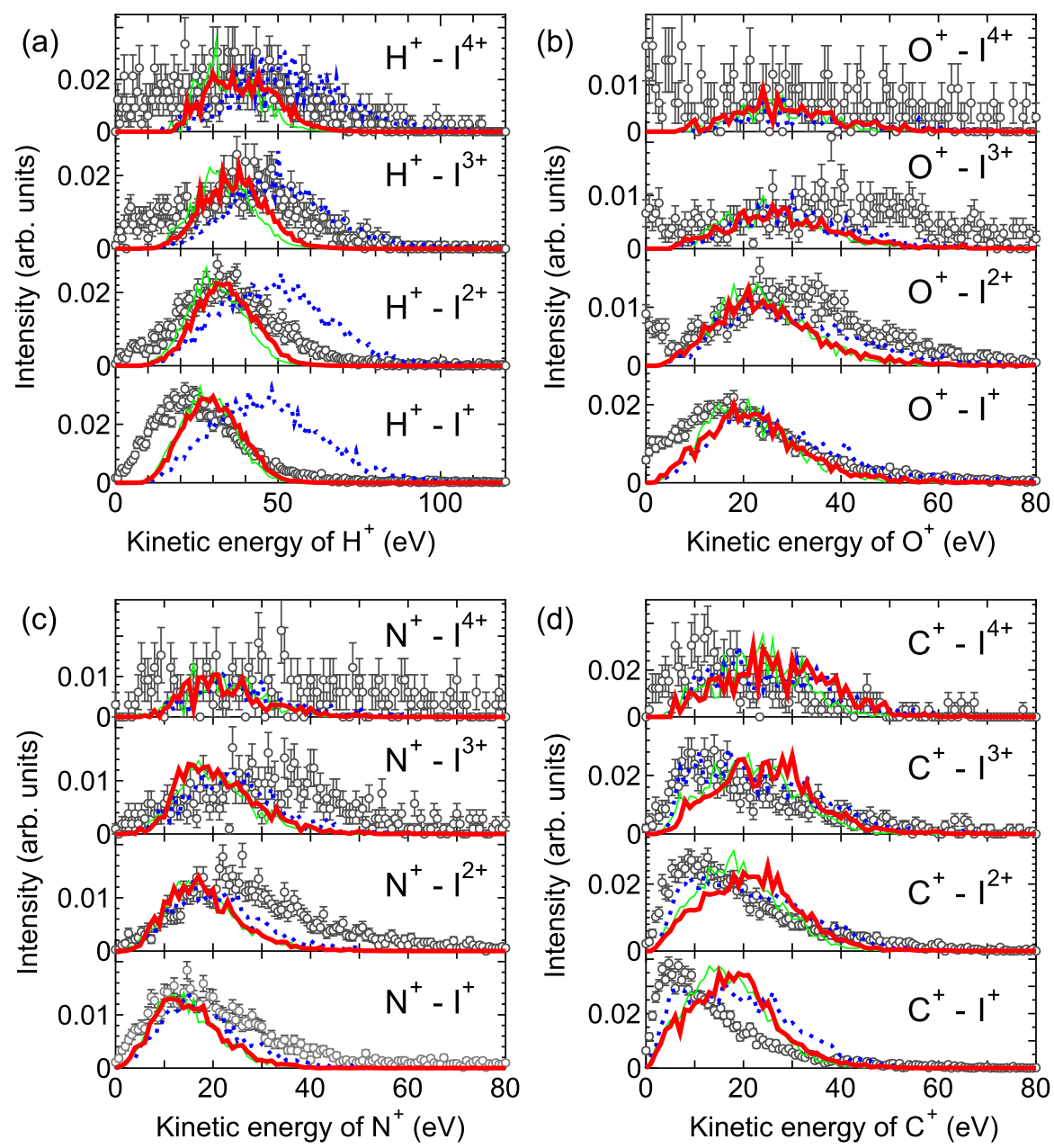

FIG. 2. Kinetic energy distribution (KED) of fragment ions. (a) KED of $\mathrm{H}^{+}$emitted from a XFEL irradiated 5-iodouracil and detected in coincidence with iodine ions. (b) KED of $\mathrm{O}^{+}$, (c) $\mathrm{KED}$ of $\mathrm{N}^{+}$, and (d) KED of $\mathrm{C}^{+}$. Symbols are the experimental data, and lines are the result of MD simulations at $T=300 \mathrm{~K}$. Dotted blue lines and thin solid green lines correspond to charge buildup times $\tau=0$ and $10 \mathrm{fs}$, respectively, assuming that the charge redistribution is instantaneous. The thick red lines represent a modeling with $\tau=10 \mathrm{fs}$ and $R=0.5 \mathrm{fs}^{-1}$. 
[31]. Indeed, taking into account a realistic charge buildup time constant of $10 \mathrm{fs}$ improves the agreement dramatically (thin green solid lines in Fig. 2), especially for coincidences of $\mathrm{H}^{+}$with the low-charge states of iodine. Thus, the KEDs of $\mathrm{H}^{+}$are particularly sensitive indicators of early molecular and charge dynamics.
Finally, the charge redistribution rate constant $R$ of $0.5 \mathrm{fs}^{-1}$ was included in the most realistic model (thick red solid lines). For the heavier ions, the simulated KEDs are less sensitive to the model parameters. These ions travel only over short distances during the charge buildup, and thus most of their kinetic energy is accumulated after the
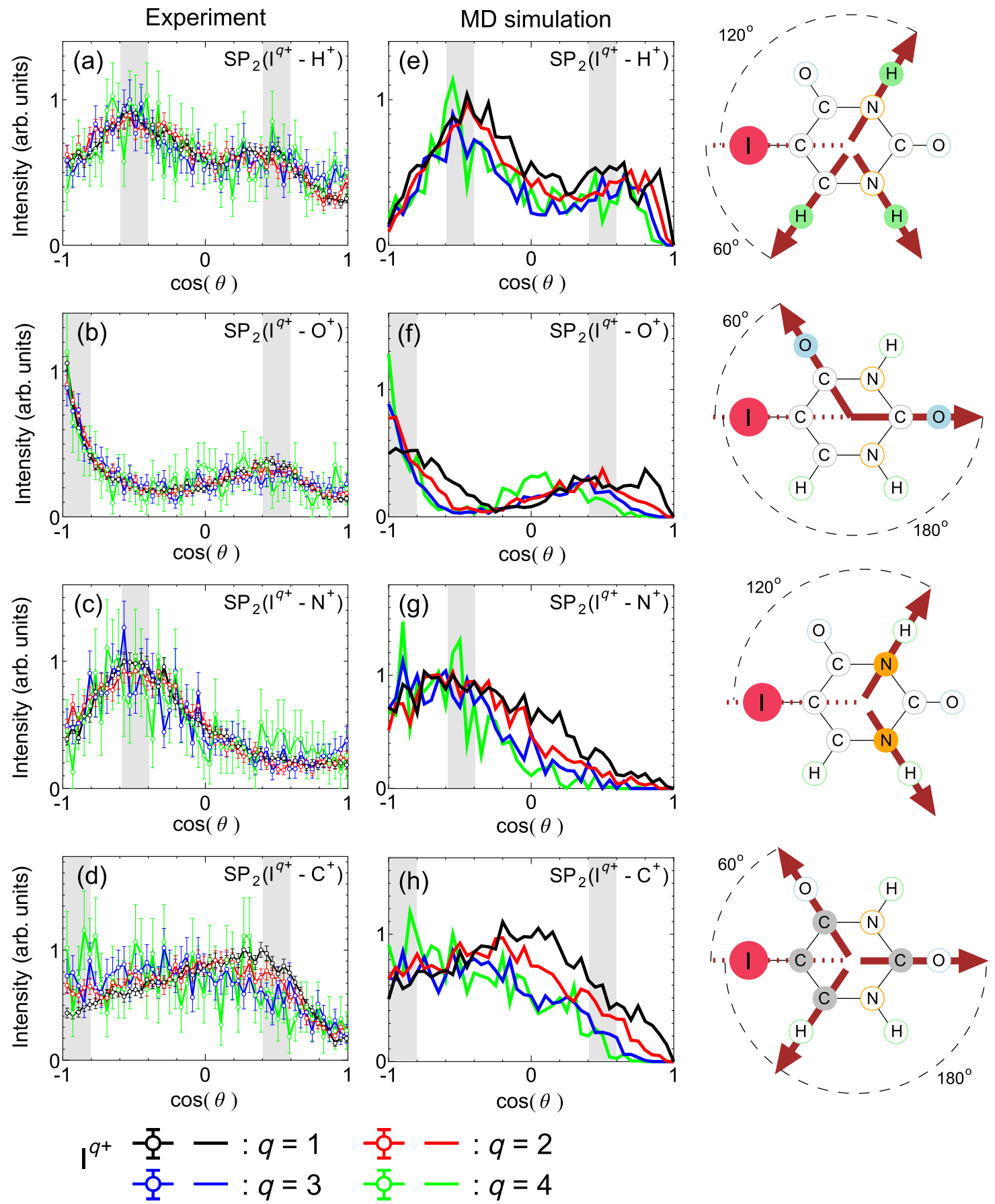

FIG. 3. Distributions of normalized scalar product of momentum vectors, $\mathrm{SP}_{2}(\mathrm{~A}, \mathrm{~B})$, plotted as a function of $\cos (\theta)$. (a) $\mathrm{SP} 2(\mathrm{~A}, \mathrm{~B})$ for the $\left(\mathrm{I}^{q+}-\mathrm{H}^{+}\right)$pair, (b) $\mathrm{SP}_{2}(\mathrm{~A}, \mathrm{~B})$ for $\left(\mathrm{I}^{q+}{ }_{-} \mathrm{N}^{+}\right)$, (c) $\mathrm{SP}_{2}(\mathrm{~A}, \mathrm{~B})$ for $\left(\mathrm{I}^{q+}{ }_{-} \mathrm{O}^{+}\right)$, and (d) $\mathrm{SP}_{2}(\mathrm{~A}, \mathrm{~B})$ for $\left(\mathrm{I}^{q+}{ }_{-} \mathrm{C}^{+}\right)$. Gray shadow areas indicate the angles of atoms in the neutral parent molecule. (e)-(h) $\mathrm{SP}_{2}(\mathrm{~A}, \mathrm{~B})$ calculated with temperature, charge buildup, and charge redistribution taken into account, using the parameters $T=300 \mathrm{~K}, \tau=10 \mathrm{fs}$, and $R=0.5 \mathrm{fs}^{-1}$. 
final charge is reached, in contrast to the $\mathrm{H}^{+}$case. Thus, it is difficult to extract information on charge dynamics from the KEDs of ions heavier than $\mathrm{H}^{+}$. The remaining discrepancies in the KEDs may come from neglecting the molecular bonds, such as initial carbon departure as dimers. Also, the smooth charge buildup model is best suited for
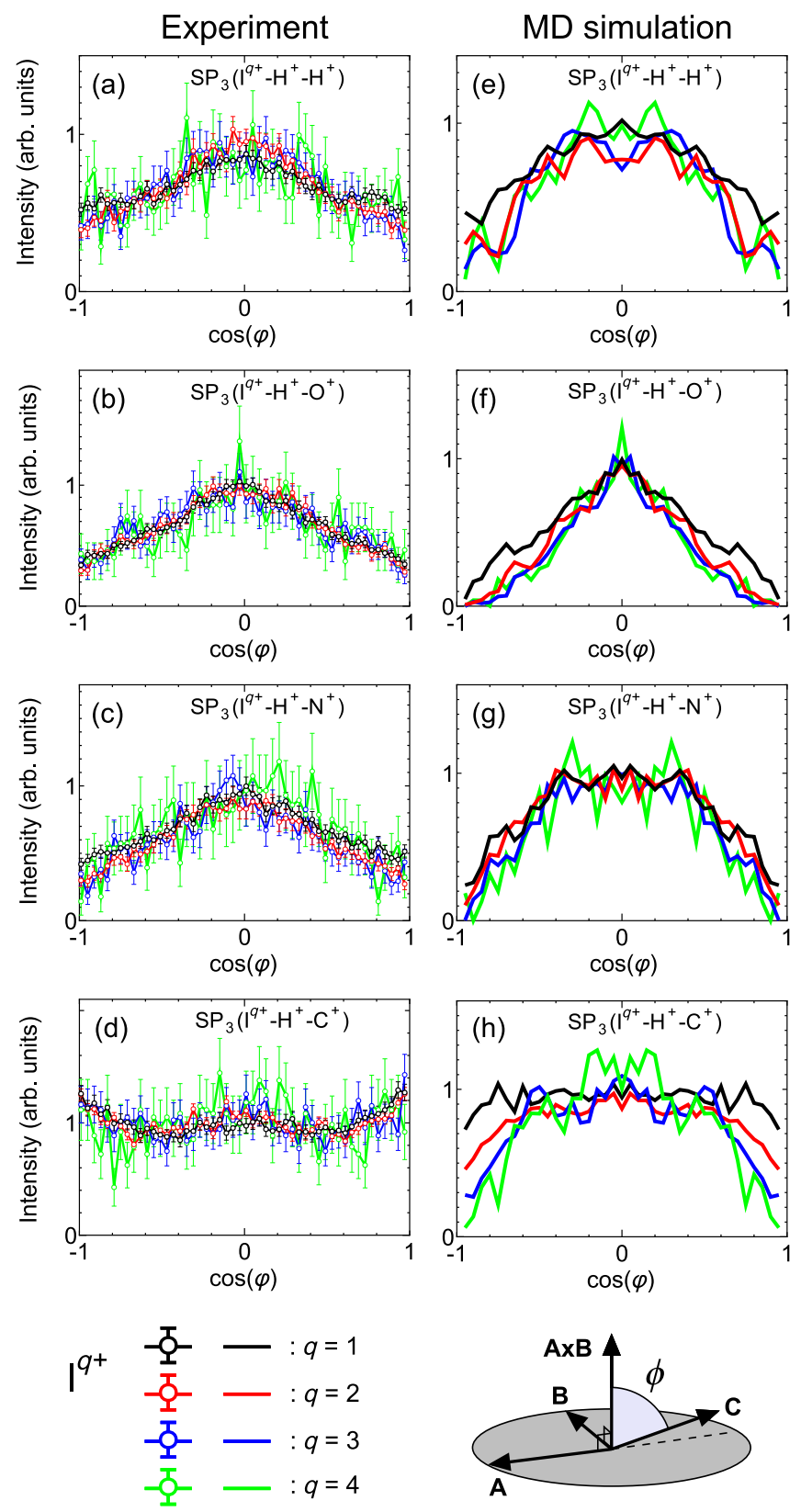

FIG. 4. Distribution of normalized scalar triplet products of momentum vectors $\mathrm{SP}_{3}(\mathrm{~A}, \mathrm{~B}, \mathrm{C})$ plotted as a function of $\cos (\phi)$. (a) $\mathrm{SP}_{3}(\mathrm{~A}, \mathrm{~B}, \mathrm{C})$ for $\left(\mathrm{I}^{q+}-\mathrm{H}^{+}-\mathrm{H}^{+}\right)$, (b) $\mathrm{SP}_{3}(\mathrm{~A}, \mathrm{~B}, \mathrm{C})$ for $\left(\mathrm{I}^{q+}-\mathrm{H}^{+}-\mathrm{O}^{+}\right)$, (c) $\mathrm{SP}_{3}(\mathrm{~A}, \mathrm{~B}, \mathrm{C})$ for $\left(\mathrm{I}^{q+}-\mathrm{H}^{+}-\mathrm{N}^{+}\right)$, and (d) $\mathrm{SP}_{3}(\mathrm{~A}, \mathrm{~B}, \mathrm{C})$ for $\left(\mathrm{I}^{q+}-\mathrm{H}^{+}-\mathrm{C}^{+}\right)$. (e)-(h) $\mathrm{SP}_{3}(\mathrm{~A}, \mathrm{~B}, \mathrm{C})$ calculated with temperature, charge buildup, and charge redistribution taken into account, using the parameters $T=300 \mathrm{~K}, \tau=10 \mathrm{fs}$, and $R=0.5 \mathrm{fs}^{-1}$. highly charged particles and can lead to discrepancies in the case of very low charge states [31]. The discrepancy between the measured and simulated KEDs is most significant for the $\mathrm{C}^{+}$case. It may be worth noting that three of the carbon atoms are close to the iodine atom, forming part of the uracil ring. Such geometrical factors neglected in the present model may also account for the discrepancy.

Previously, Motomura et al. demonstrated that the momentum correlations are well suited for the reconstruction of early molecular dynamics [31]. Simulated $\mathrm{SP}_{2}(\mathrm{~A}, \mathrm{~B})$ and $\mathrm{SP}_{3}(\mathrm{~A}, \mathrm{~B}, \mathrm{C})$ are displayed in the right-hand side of Figs. 3 and 4 , respectively. We found good agreement between the experiment and MD results with $T=300 \mathrm{~K}, \tau=10 \mathrm{fs}$, and $R=0.5 \mathrm{fs}^{-1}$ (see Ref. [39] for many more parameter combinations). These values are close to the ones for $\mathrm{CH}_{3} \mathrm{I}$ ( $\tau=9$ fs and $R=0.37 \mathrm{fs}^{-1}$ [31]), suggesting that the model employed is indeed of general applicability. It may be worth noting also that these values of $R$ are comparable to the Auger decay rates of individual decay stages in Xe, as calculated by Son and Santra [40]. The details of parameter dependence of $\mathrm{SP}_{2}(\mathrm{~A}, \mathrm{~B})$ and $\mathrm{SP}_{3}(\mathrm{~A}, \mathrm{~B}, \mathrm{C})$ are presented in Ref. [39], but it is worth pointing out here that the internal temperature also strongly affects the correlated motion of the particles in the molecular Coulomb explosion.

Our results from both experiment and modeling show that there is a clear difference in the behavior of carbon ions compared to oxygen, nitrogen, and also hydrogen ions. All the latter exhibit a good angular correlation with the iodine's momentum that is consistent with the initial geometry of 5-IU (Fig. 3); they also show a marked tendency of planar emission together with $\mathrm{I}^{q+}$ and $\mathrm{H}^{+}$ (Fig. 4). Hydrogen and oxygen ions, being attached to the ring, experience mostly radial forces. Also, the nitrogen ions experience outward, mostly planar, forces after the early departure of protons. Carbon ions, however, are surrounded by elements of equal or larger mass, and the forces on some of the carbon ions, such as $\mathrm{C}_{1}$ and $\mathrm{C}_{2}$, are not necessarily outwards. Such a typical example is shown in Fig. S7 of Ref. [39]. This may explain a peculiar behavior in the observed momentum correlations in which the momentum of the carbon ion is involved.

Since we validated the model by comparison with the experimental observables, we can now use it to give insights into motions of the individual atoms in real space that may deteriorate $\mathrm{x}$-ray scattering signals necessary for the structure retrieval of the original object with XFEL. The simulated time evolution of interatomic distances using realistic charge dynamics is displayed in Fig. 5. The results are an average of over 1000 runs for each $Q^{\max }$, also weighted by the charge state distribution of parent molecular ions, estimated from our $\mathrm{CH}_{3} \mathrm{I}$ experiment [31]. MD results reveal that after 10 fs (the XFEL pulse duration), the $\mathrm{H}-\mathrm{C}$ and $\mathrm{H}-\mathrm{N}$ distances increase by $50 \%-100 \%$, whereas the increases of the O-C, C-N, and C-C bonds are of only a few percent; it takes more than 10 fs for heavy atoms, 


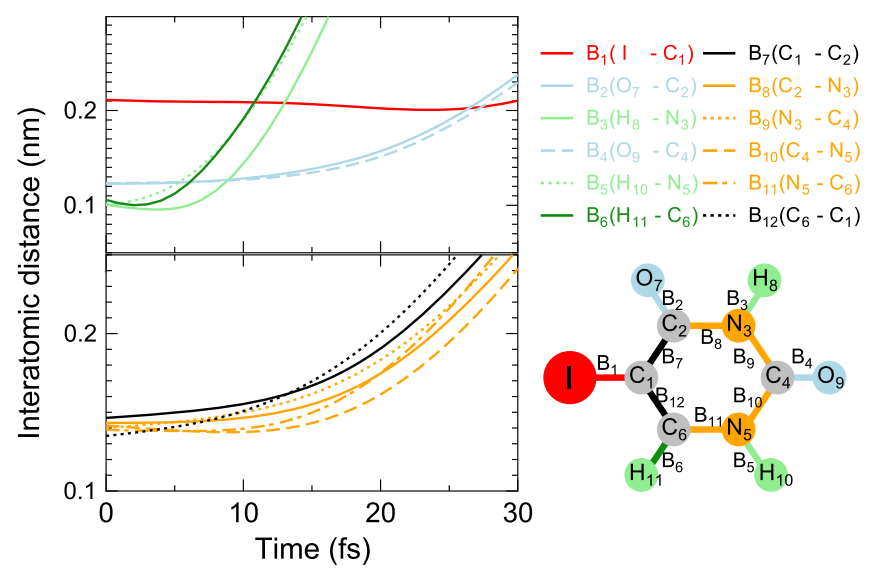

FIG. 5. Time evolution of interatomic distances in 5-IU obtained by MD simulations. Upper panel: Interatomic distance of I-C (red line), O-C (sky-blue lines), H-C (dark-green line), and $\mathrm{H}-\mathrm{N}$ (light-green lines) pairs. Lower panel: Interatomic distance of C-C (black lines) and $\mathrm{C}-\mathrm{N}$ (orange lines) pairs.

except for $\mathrm{H}$, to move $10 \mathrm{pm}$. The smallest change takes place in the C-I distance and is due to the large mass of iodine and the fact that the Coulomb forces acting on $\mathrm{C}_{1}$ tend to cancel out at the early stages of the explosion. These changes are much smaller than what would be expected from instantaneous charge creation (Ref. [39], Fig. S7).

\section{CONCLUSION}

We carried out recoil-ion momentum imaging measurements for 5-iodouracil (IU) using intense XFEL pulses at SACLA. The parametric, classical Coulomb explosion model, which includes both charge and nuclear dynamics, succeeded in reproducing the experimental observations. We found that both the charge buildup and redistribution take place within the XFEL pulse duration of $10 \mathrm{fs}$, at the early stages of the Coulomb explosion. Good agreement between experiment and MD results is found when selecting an internal temperature, charge buildup time, and charge redistribution rate of $300 \mathrm{~K}, 10 \mathrm{fs}$, and $0.5 \mathrm{fs}^{-1}$, respectively. The present values of charge buildup time $\tau$ and charge redistribution rate $R$ are very close to those we obtained for $\mathrm{CH}_{3} \mathrm{I}$ ( $\tau=9$ fs and $R=0.37 \mathrm{fs}^{-1}$ [31]). The time dependence of the interatomic distances, deduced from the MD simulations, suggests that within $10 \mathrm{fs}$, the displacements are large for the hydrogens and are considerably smaller for the other heavier atoms. This result, obtained on a realistic, biologically relevant molecule, gives two important indications, which, being independent of the exact composition of the studied nucleobase, are both of very general relevance to other such molecules: (i) If short enough XFEL pulses are used, the single-shot coherent diffraction imaging approach of single bio-macromolecules will interrogate a target sample left practically unperturbed, and (ii) the molecular basis of the radiosensitizing action of 5-IU might be related to the local production of a "radiation soup" consisting of relatively energetic ions whose local damage effect adds to that of the genotoxic low-energy electrons generated by electronic relaxation cascading mechanisms. The new mechanism revealed here generally contributes to the understanding of how radiosensitizers work and should inspire therapists in the design of novel radiosensitizing drugs. Last, but not least, let us point out that these are the first "time-resolved" results characterizing the very early dynamics of a nucleobase in interaction with an intense XFEL pulse, using a multiparticle coincidence technique combined with MD simulations. This study proposes a complementary approach to the challenging true pump-probe experiment, with the addition of high time resolution and high-quality statistical data. Moreover, the modeling proposed has a good potential to be generally applicable to other systems of this kind.

\section{ACKNOWLEDGMENTS}

The experiments were performed at SACLA with the approval of JASRI and the program review committee (No. 2012B8045). This study was supported by the X-ray Free Electron Laser Utilization Research Project and the X-ray Free Electron Laser Priority Strategy Program of the Ministry of Education, Culture, Sports, Science and Technology of Japan (MEXT), by the Japan Society for the Promotion of Science (JSPS), by the Proposal Program of SACLA Experimental Instruments of RIKEN, and by the IMRAM project. E. K. acknowledges support by the Academy of Finland, and Y. H. J. by the National Basic Research Program of China (No. 2013CB922200), the Natural Science Foundation of China (No. 11420101003, No. 11274232, and No. 61308068). A. R. was supported by the Chemical Sciences, Geosciences, and Biosciences Division of the Office of Basic Energy Sciences, Office of Science, U.S. Department of Energy under Contract No. DE-FG02-86ER1349. A. M. and D. E. K. acknowledge the Global Research Laboratory Program (No. 200900439) and the Max Planck POSTECH/KOREA Research Initiative Program (No. 2011-0031558) through the National Research Foundation of Korea (NRF) funded by Ministry of Science, ICT \& Future Planning. J. H. C. acknowledges support by the Shanghai Natural Science Foundation (No. 13ZR1464700) and the Knowledge Innovation Project of the Chinese Academy of Sciences (No. 255015061).

\section{APPENDIX: MATERIALS AND METHODS}

\section{Experimental methods}

The experiment was carried out at the experimental hutch 3 (EH3) of beam line 3 (BL3) of SACLA [41]. The XFEL beam is focused by the Kirkpatrick-Baez (KB) 
mirror system to a focal size of $1 \mu \mathrm{m}$ (FWHM) in diameter [42].

The photon energy was set at $5.5 \mathrm{keV}$ and the photon bandwidth was about $60 \mathrm{eV}$ (FWHM). The repetition rate of the XFEL pulses was $10 \mathrm{~Hz}$. The pulse length was not measured but was estimated to be about 10 fs (FWHM) [43]. XFEL pulse energies were measured by the beamposition monitor [44] located upstream of the beam line. The monitor was calibrated by a calorimeter [45] so that output signals from the monitor could be transformed into the absolute value of the pulse energy, which was $235 \mu \mathrm{J}$ on average. The relative $\mathrm{x}$-ray pulse energy passing through the interaction point was also measured shot-to-shot by a p-intrinsic-n (PIN) photodiode. The shot-to-shot pulse energy fluctuation was about $7 \%$ (14\% FWHM). The peak fluence was $26 \mu \mathrm{J} / \mu \mathrm{m}^{2}$ on average. The absolute value of the peak fluence was calibrated just after the experiment using Ar [27].

The 5-iodouracil ( $>99.0 \%)$ was purchased from Tokyo Chemical Industry Co., Ltd. and used without further purification. The solid sample was heated up to $190^{\circ} \mathrm{C}$ in the reservoir of a modified electromagnetic solenoid valve [46]. The resulting sample vapor was seeded by helium gas and introduced to the focal point of the XFEL pulses in the ultrahigh-vacuum reaction chamber as a pulsed supersonic gas jet. Any change of sample due to heating was not confirmed by ${ }^{1} \mathrm{H}$ NMR measurements.

The ions were detected by a multicoincidence recoil ion momentum spectrometer $[36,37]$ to measure threedimensional momenta of each fragment ion. The molecular beam was crossed with the focused XFEL beam at the reaction point, and the emitted ions were projected by electrostatic fields onto an 80-mm-diameter microchannel plate (MCP) detector, in front of a delay-line anode. The three-dimensional momentum vectors were determined using both the TOF and the detector-hit position for each ion. A three-layer-type delay-line anode (Roentdek HEX80) was used to minimize the dead time between hits [36]. The design of the ion spectrometer was similar to the one described in Ref. [37], but the electrodes were modified to enable detection of high-energy ions. The distance between the reaction point and HEX80 was $228 \mathrm{~mm}$. The electrostatic gradient field was produced by five electrodes, labeled U3, U2, U1, D1, and D2. The maximum acceptable kinetic energy of our spectrometer was about $80 \mathrm{q} \mathrm{eV}$ for the multiply charged ion with q charge when the voltages of $8.0,7.36,6.4,4.56$, and $0 \mathrm{kV}$ were applied to the U3, U2, U1, D1, and D2 electrodes, respectively.

The observed particle hit rate was kept at less than 0.1 events per FEL shot during experiments. False coincidence was negligible for the coincidence analysis.

\section{MD simulations}

Classical molecular dynamics simulations were carried out, where we evaluated the Coulomb repulsive force between ions during ion trajectory calculations, while the effects of covalent bonds in the 5-IU molecule were neglected. We traced the trajectories of the emitted ions to up to $10 \mathrm{ps}$ with a time step of $0.2 \mathrm{fs}$. The ensemble of initial positions and velocities of atoms in the parent molecules was prepared by B3LYP/3-21G(d) quantumchemical calculations using the GAMESS package [47]. Thermal vibration effects were taken into account using Nose-Hoover thermostat methods implemented in the GAMESS package.

For each trajectory of the MD simulations, the final charge state of parent 5-IU molecules, $Q^{\max }$, was chosen according to the experimental charge distribution of the $\mathrm{CH}_{3} \mathrm{I}$ molecules [31] that was measured under the same XFEL conditions. We also determined the final charge of each atom in such a way that the charges are randomly distributed over the atoms in the parent molecule. In addition, we assumed that the iodine ions take at least +1 charge. The time evolution of the atomic charges was governed by the equations given in Sec. III. We carried out numerous MD simulations by scanning the parameters $\tau, R$, and $T$, as presented in Ref. [39].

[1] W. Ackermann et al., Operation of a Free-Electron Laser from the Extreme Ultraviolet to the Water Window, Nat. Photonics 1, 336 (2007).

[2] P. Emma et al., First Lasing and Operation of an AngstromWavelength Free-Electron Laser, Nat. Photonics 4, 641 (2010).

[3] T. Ishikawa et al., A Compact X-ray Free-Electron Laser Emitting in the Sub-ångström Region, Nat. Photonics 6, 540 (2012).

[4] B. Erk et al., Imaging Charge Transfer in Iodomethane upon X-ray Photoabsorption, Science 345, 288 (2014).

[5] P. Beaud et al., A Time-Dependent Order Parameter for Ultrafast Photoinduced Phase Transitions, Nat. Mater. 13, 923 (2014).

[6] R. Mankowsky et al., Nonlinear Lattice Dynamics as a Basis for Enhanced Superconductivity in $\mathrm{YBa}_{2} \mathrm{Cu}_{3} \mathrm{O}_{6.5}$, Nature (London) 516, 71 (2014).

[7] S. E. Canton et al., Visualizing the Non-equilibrium Dynamics of Photoinduced Intramolecular Electron Transfer with Femtosecond X-ray Pulses, Nat. Commun. 6, 6359 (2015).

[8] K. H. Kim et al., Direct Observation of Bond Formation in Solution with Femtosecond X-ray Scattering, Nature (London) 518, 385 (2015).

[9] T. R. M. Barends et al., Direct Observation of Ultrafast Collective Motions in CO Myoglobin upon Ligand Dissociation, Science 350, 445 (2015).

[10] C. E. Liekhus-Schmaltz et al., Ultrafast Isomerization Initiated by X-ray Core Ionization, Nat. Commun. 6, 8199 (2015).

[11] Ph. Wernet et al., Orbital-Specific Mapping of the Ligand Exchange Dynamics of $\mathrm{Fe}(\mathrm{CO})_{5}$ in Solution, Nature (London) 520, 78 (2015). 
[12] H. Öström et al., Probing the Transition State Region in Catalytic CO Oxidation on Ru, Science 347, 978 (2015).

[13] Y. Uemura et al., Dynamics of Photoelectrons and Structural Changes of Tungsten Trioxide Observed by Femtosecond Transient XAFS, Angew. Chem., Int. Ed. Engl. 55, 1364 (2016).

[14] K. R. Ferguson et al., Transient Lattice Compression in the Solid-to-Plasma Transition, Sci. Adv. 2, e1500837 (2016).

[15] M. M. Seibert et al., Single Mimivirus Particles Intercepted and Imaged with an X-ray Laser, Nature (London) 470, 78 (2011).

[16] T. Kimura et al., Imaging Live Cell in Micro-liquid Enclosure by X-ray Laser Diffraction, Nat. Commun. 5, 3052 (2014).

[17] L. F. Gomez et al., Shapes and Vorticities of Superfluid Helium Nanodroplets, Science 345, 906 (2014).

[18] Y. Kang et al., Crystal Structure of Rhodopsin Bound to Arrestin by Femtosecond X-ray Laser, Nature (London) 523, 561 (2015).

[19] K. Ayyer et al., Macromolecular Diffractive Imaging Using Imperfect Crystals, Nature (London) 530, 202 (2016).

[20] R. Neutze, R. Wouts, D. van der Spoel, E. Weckert, and J. Hajdu, Potential for Biomolecular Imaging with Femtosecond X-ray Pulses, Nature (London) 406, 752 (2000).

[21] R. W. Howell, Auger Processes in the 21st Century, Int. J. Radiat. Biol. 84, 959 (2008).

[22] K. Gokhber, P. Kolorenč, A. I. Kuleff, and L. S. Cederbaum, Site- and Energy-Selective Slow-Electron Production through Intermolecular Coulombic Decay, Nature (London) 505, 661 (2014).

[23] F. Trinter et al., Resonant Auger Decay Driving Intermolecular Coulombic Decay in Molecular Dimers, Nature (London) 505, 664 (2014).

[24] L. Young et al., Femtosecond Electronic Response of Atoms to Ultra-Intense X-rays, Nature (London) 466, 56 (2010).

[25] G. Doumy et al., Nonlinear Atomic Response to Intense Ultrashort X rays, Phys. Rev. Lett. 106, 083002 (2011).

[26] B. Rudek et al., Ultra-efficient Ionization of Heavy Atoms by Intense X-ray Free-Electron Laser Pulses, Nat. Photonics 6 , 858 (2012).

[27] H. Fukuzawa et al., Deep Inner-Shell Multiphoton Ionization by Intense X-ray Free-Electron Laser Pulses, Phys. Rev. Lett. 110, 173005 (2013).

[28] T. Gorkhover et al., Nanoplasma Dynamics of Single Large Xenon Clusters Irradiated with Superintense X-ray Pulses from the Linac Coherent Light Source Free-Electron Laser, Phys. Rev. Lett. 108, 245005 (2012).

[29] T. Tachibana et al., Nanoplasma Formation by High Intensity Hard X-rays, Sci. Rep. 5, 10977 (2015).

[30] B. Erk et al., Ultrafast Charge Rearrangement and Nuclear Dynamics upon Inner-Shell Multiple Ionization of Small Polyatomic Molecules, Phys. Rev. Lett. 110, 053003 (2013).

[31] K. Motomura et al., Charge and Nuclear Dynamics Induced by Deep Inner-Shell Multiphoton Ionization of $\mathrm{CH}_{3} \mathrm{I}$
Molecules by Intense X-ray Free-Electron Laser Pulses, J. Phys. Chem. Lett. 6, 2944 (2015).

[32] C. Heidelberger, N. K. Chaudhuri, P. Danneberg, D. Mooren, L. Griesbach, R. Duschinsky, R. J. Schnitzer, E. Pleven, and J. Scheiner, Fluorinated Pyrimidines, a New Class of Tumour-Inhibitory Compounds, Nature (London) 179, 663 (1957).

[33] B. A. Kihlman, The Effect of 5-halogenated deoxyuridines on the Frequency of X-ray-Induced Chromosomal Aberrations in Vica Faba, Hereditas 49, 353 (1963).

[34] E. Pomplun and G. Sutmann, Is Coulomb Explosion a Damaging Mechanism for ${ }^{125} \mathrm{IUdR}$ ?, Int. J. Radiat. Biol. 80, 855 (2004).

[35] E. A. Kümmerle and E. Pomplun, Charge Build-up During Decay of DNA-incorporated ${ }^{123 / 125} \mathrm{I}$ : Consequences for Labeled Molecular Structures, Int. J. Radiat. Biol. 88, 922 (2012).

[36] O. Jagutzki et al., Multiple Hit Readout of a Microchannel Plate Detector with a Three-Layer Delay-Line Anode, IEEE Trans. Nucl. Sci. 49, 2477 (2002).

[37] H. Fukuzawa et al., Electron Spectroscopy of Rare-Gas Clusters Irradiated by X-ray Free-Electron Laser Pulses from SACLA, J. Phys. B 49, 034004 (2016).

[38] V. Stumpf, K. Gokhberg, and L. S. Cederbaum, The Role of Metal Ions in X-ray-Induced Photochemistry, Nat. Chem. 8, 237 (2016).

[39] See Supplemental Material at http://link.aps.org/ supplemental/10.1103/PhysRevX.6.021035 for the results of classical MD simulations of the Coulomb explosion model by various parameter combinations.

[40] S.-K. Son and R. Santra, Monte Carlo Calculation of Ion, Electron, and Photon Spectra of Xenon Atoms in X-ray Free-Electron Laser Pulses, Phys. Rev. A 85, 063415 (2012).

[41] K. Tono et al., Beamline, Experimental Stations and Photon Beam Diagnostics for the Hard X-ray Free Electron Laser of SACLA, New J. Phys. 15, 083035 (2013).

[42] H. Yumoto et al., Focusing of X-ray Free-Electron Laser Pulses with Reflective Optics, Nat. Photonics 7, 43 (2013).

[43] Y. Inubushi et al., Determination of the Pulse Duration of an $X$-ray Free Electron Laser Using Highly Resolved SingleShot Spectra, Phys. Rev. Lett. 109, 144801 (2012).

[44] K. Tono, T. Kudo, M. Yabashi, T. Tachibana, Y. Feng, D. Fritz, J. Hastings, and T. Ishikawa, Single-Shot BeamPosition Monitor for X-ray Free Electron Laser, Rev. Sci. Instrum. 82, 023108 (2011).

[45] M. Kato et al., Pulse Energy Measurement at the Hard X-ray Laser in Japan, Appl. Phys. Lett. 101, 023503 (2012).

[46] T. Ebata, Study on the Structure and Vibrational Dynamics of Functional Molecules and Molecular Clusters by Double Resonance Vibrational Spectroscopy, Bull. Chem. Soc. Jpn. 82, 127 (2009).

[47] M. W. Schmidt et al., General Atomic and Molecular Electronic Structure System, J. Comput. Chem. 14, 1347 (1993). 